\title{
Real World Comparison of Rivaroxaban and Warfarin in Korean Patients with Atrial Fibrillation: Propensity Matching Cohort Analysis
}

\author{
Hyung Ki Jeong ${ }^{\dagger}$, Ki Hong Lee ${ }^{\dagger}$, Hyung Wook Park, Nam Sik Yoon, Min Chul Kim, Nuri Lee, \\ Ji Sung Kim, Youngkeun Ahn, Myung Ho Jeong, Jong Chun Park, and Jeong Gwan Cho* \\ Department of Cardiovascular Medicine, Chonnam National University Hospital, Gwangju, Korea
}

Rivaroxaban has emerged as a potential alternative to warfarin for the prevention of thromboembolism in patients with atrial fibrillation (AF). However, there has been concern for the risk of major bleeding, especially in Asian patients. We investigated the efficacy and safety of rivaroxaban compared to warfarin in Korean real world practice. A total of 2,208 consecutive non-valvular AF patients were divided into the Warfarin group $(\mathrm{n}=990)$ and the Rivaroxaban group $(\mathrm{n}=1218)$. Propensity matched 1 -year clinical outcomes were compared (Warfarin, $\mathrm{n}=804$; Rivaroxaban, $\mathrm{n}=804$ ). The efficacy outcome was defined as stroke/systemic embolism (SE). The safety outcome was major bleeding. The primary net clinical benefit (NCB) was defined as the composite of stroke/SE, major bleeding, and all-cause mortality. Secondary, NCB was defined as the composite of stroke, $\mathrm{SE}$, and major bleeding. Rivaroxaban had the similar efficacy in terms of thromboembolic event prevention [hazard ratio (HR) $0.69,95 \%$ confidence interval (CI) 0.37-1.32, $\mathrm{p}=0.266$ ] compared to warfarin. Rivaroxaban significantly lowered the risk of major bleeding [HR $0.41,95 \%$ CI $0.22-0.76, p=0.004]$. Primary NCB was significantly low in the rivaroxaban group [HR 0.54, 95\% CI 0.36-0.81, $\mathrm{p}=0.003$ ]. Secondary NCB was also low in the rivaroxaban group [HR 0.62, 95\% CI 0.40-0.99, $\mathrm{p}=0.041]$. Both rivaroxaban $15 \mathrm{mg}$ and $20 \mathrm{mg}$ groups had similar efficacy and significantly lower risks of major bleeding as well as primary and secondary NCB compared to the warfarin group. In patients with non-valvular AF, rivaroxaban had a similar efficacy to warfarin in Korean real world practice. However, rivaroxaban had better safety and net clinical outcomes compared to warfarin.

\section{Key Words: Atrial Fibrillation; Rivaroxaban; Warfarin; Thromboembolism; Hemorrhage}

This is an Open Access article distributed under the terms of the Creative Commons Attribution Non-Commercial License (http://creativecommons.org/licenses/by-nc/4.0) which permits unrestricted non-commercial use, distribution, and reproduction in any medium, provided the original work is properly cited.

\section{Article History:}

Received December 14, 2018

Revised December 31, 2018

Accepted January 2, 2019

\section{Corresponding Author:}

Jeong Gwan Cho

Department of Cardiovascular

Medicine, Chonnam National

University Hospital, 42 Jaebong-ro,

Dong-gu, Gwangju 61469, Korea

Tel: +82-62-220-6242

Fax: +82-62-223-3105

E-mail:drgood2@empas.com

${ }^{\dagger}$ These authors contributed equally to this work.

\section{INTRODUCTION}

Atrial fibrillation (AF) is well known to increase the risk of stroke, systemic thromboembolic events significantly, and it has a twofold risk for all-cause mortality. ${ }^{1}$ Therefore, oral anticoagulants (OACs) like vitamin $\mathrm{K}$ antagonists are indicated to prevent strokes and systemic embolism in patients with $\mathrm{AF}$ who have more than 1 point on the $\mathrm{CHA}_{2} \mathrm{DS}_{2}$-VASc score. ${ }^{2}$ However, there always have been concerns of bleeding risks for those taking OACs. Recently, non-vitamin K antagonist oral anticoagulants (NOACs) have emerged as promising alternatives to warfarin for comparable thromboembolic events prevention with reducing the risk of major bleeding. Nonetheless, NOACs had concerns about increased risk of gastrointestinal (GI) bleeding. ${ }^{3-6}$ However, consequent real world data were inconsistent with previous RCTs in terms of GI bleeding which also demonstrated a lower rate in NOACs. ${ }^{7,8}$

For Asian patients, it is difficult to maintain an International normalized ratio (INR) within target therapeutic 
range of 2 to 3 . Furthermore, intracranial hemorrhage (ICH) occurred even in the patients who were generally within the target therapeutic INR range. ${ }^{9}$ Most relevant independent risk factor for ICH was ethnic group as Asian. Thus, Asian patients have far higher risks for intracranial hemorrhage than non-Asian patients when they took warfarin. $^{10,11}$ A comparison between Asians and nonAsians in the subgroup analysis from 4 pivotal NOACs trials demonstrated that NOACs had better efficacy for the prevention of stroke and bleeding risk in Asian than non-Asian patients. ${ }^{12,13}$ Therefore, NOACs might be more attractive treatment options for Asian AF patients. Consequently, several large scale real-world studies of NOACs also support better efficacy and safety of NOACs compared to warfarin in Asian AF patients. ${ }^{714-17}$ Nonetheless, there have been few studies in Korean non-valvular AF patients. Therefore, we investigated the efficacy and safety of rivaroxaban compared to warfarin in Korean real world practice.

\section{MATERIALS AND METHODS}

\section{Study population}

A total of 2,208 consecutive, non-valvular AF patients were enrolled between January 2014 and December 2016 at the department of Neurology and Cardiology at Chonnam National University Hospital in Gwangju, South Korea. They were divided into 2 groups which were the Warfarin group $(\mathrm{n}=990,44.8 \%)$ and the Rivaroxaban group ( $\mathrm{n}=1218,55.2 \%)$. The inclusion criteria were patients who were taking OACs whether warfarin or Rivaroxaban and having more than 2 points on the $\mathrm{CHA}_{2} \mathrm{DS}_{2}$-VASc score. The exclusion criteria were valvular $\mathrm{AF}$ (rheumatic mitral stenosis, prosthetic mitral valve replacement or mitral valve repair), or any OAC class change (from warfarin to NOACs, from NOACs to warfarin). After a propensity score (PS) matching to have the similar baseline characteristics, the patient groups were 1:1 matched (Warfarin, $\mathrm{n}=804$; Rivaroxaban, $\mathrm{n}=804$ ). The follow-up period was 1 year or until the first occurrence of any study outcomes since the enrollment. The study was approved by the ethics committee at Chonnam National University Hospital, Gwangju, South Korea (CNUH-2018-109), where informed consent was exempted from enrolled patients because of the retrospective study protocol.

\section{Definition}

Stroke was defined as the sudden onset of focal neurologic deficit which was consistent with the territory of a major cerebral artery. It was classified as an ischemic, hemorrhagic, or transient ischemic attack (TIA). TIA was defined as the sudden onset of a focal neurologic defect without definitive evidence of newly detected cerebral lesions. A systemic embolism (SE) was defined as an acute vascular occlusion of any major artery which was detected by imaging tools or surgery. Major bleeding was defined according to the International Society on Thrombosis and Hemostasis
(ISTH) criteria, as the decrease of hemoglobin levels by a $2 \mathrm{~g} / \mathrm{dL}$ or the requirement of a transfusion of more than 2 units of packed red blood cells, occurring at a critical site, or leading to death. Minor bleeding was defined as the overt bleeding which was not satisfied with the major bleeding criteria. ${ }^{18}$ The efficacy outcome was defined as stroke/SE. The safety outcome was major bleeding. Mucosal bleeding was defined as any bleeding from mucosa including gastrointestinal (GI) tract, genitourinary tract, and respiratory mucosa. Primary net clinical benefit (NCB) was defined as the composite of stroke/systemic embolism, major bleeding and all-cause mortality. Secondary NCB was defined as the composite of stroke, systemic embolism and major bleeding. The glomerular filtration rate (GFR) based on serum concentrations of creatinine was estimated using the Simplified Modification of Diet in Renal Disease formula (MDRD).

\section{Statistical analysis}

Continuous variables were described as mean value \pm SD. The student t-test and Mann-Whitney test were used to evaluate the differences for continuous variables. Discrete variables were presented as percentages and frequencies. They were analyzed using the chi-square test or Fisher's exact test between groups as appropriate. Propensity score matching was carried out to have the same baseline characteristics in each group. Propensity scores were calculated using multivariable logistic regression incorporating frequently used variables and potential risk factors including age, sex, hypertension, diabetes mellitus, previous history of stroke or TIA, previous myocardial infarction (MI), or previous history of heart failure. Matching was performed with a greedy matching protocol (1:1 nearest neighbor matching without replacement). The probability of clinical outcomes and net clinical benefits were estimated by the Kaplan-Meier method and curves were compared with the log-rank test. Comparison of efficacy, safety outcomes, and net clinical benefit were adjusted using Cox proportional hazards models. In all statistical tests, a two-sided p value $<0.05$ was considered statistically significant. Statistical analysis was performed using SPSS 21.0 for Windows (SPSS, Inc., Chicago, Illinois, USA).

\section{RESULTS}

\section{Baseline clinical characteristics}

Before propensity score matching, patients taking rivaroxaban were older and had higher prevalence of female patient, hypertension, myocardial infarction, heart failure, and a history of stroke/TIA. The mean $\mathrm{CHA}_{2} \mathrm{DS}_{2}$-VASc score was higher in patients with rivaroxaban ( $3.34 \pm 1.9$ vs. $3.5 \pm 1.7, \mathrm{p}=0.019$ ) than in patients with warfarin. The laboratory data in patients with rivaroxaban showed higher value of Hs-CRP, NT-proBNP than patients with warfarin. There was no significant difference in creatinine level between the two groups, whereas creatinine clearance was lower in patients with rivaroxaban (Table 1).

After propensity matching, there was no difference in gen- 
TABLE 1. Baseline clinical characteristics

\begin{tabular}{|c|c|c|c|c|c|c|}
\hline & \multicolumn{3}{|c|}{ Before PS matching } & \multicolumn{3}{|c|}{ After PS matching } \\
\hline & $\begin{array}{c}\text { Patients with } \\
\text { warfarin }(\mathrm{n}=990)\end{array}$ & $\begin{array}{c}\text { Patients with } \\
\text { Rivaroxaban }(\mathrm{n}=1218)\end{array}$ & p-value & $\begin{array}{c}\text { Patients with } \\
\text { warfarin }(\mathrm{n}=804)\end{array}$ & $\begin{array}{c}\text { Patients with } \\
\text { Rivaroxaban }(\mathrm{n}=804)\end{array}$ & p-value \\
\hline Female gender, $\mathrm{n}(\%)^{*}$ & $338(34.1)$ & $546(44.8)$ & $<0.001$ & $318(39.6 \%)$ & $295(36.7)$ & 0.238 \\
\hline Age, yrs & $69.3 \pm 10.8$ & $72.5 \pm 9.8$ & 0.004 & $70.4 \pm 10.2$ & $71.4 \pm 10.5$ & 0.086 \\
\hline$\geq 65$ years old & $710(71.7)$ & $977(80.2)$ & $<0.001$ & $606(75.4)$ & $605(75.2)$ & 0.954 \\
\hline \multicolumn{7}{|l|}{ Medical history, $\mathrm{n}(\%)^{*}$} \\
\hline Hypertension & $517(52.2)$ & $767(63.0)$ & $<0.001$ & $440(54.7)$ & $430(53.5)$ & 0.617 \\
\hline Diabetes mellitus & $213(21.5)$ & $292(24.0)$ & 0.185 & $179(22.3)$ & $194(24.1)$ & 0.375 \\
\hline Smoking & $265(26.8)$ & $220(18.1 \%)$ & $<0.001$ & $189(23.5)$ & $164(20.4)$ & 0.132 \\
\hline Previous history of MI & $57(5.8)$ & $132(10.8)$ & $<0.001$ & $55(6.8)$ & $55(6.8)$ & 1.000 \\
\hline Previous history of $\mathrm{HF}$ & $91(9.2)$ & $120(9.4)$ & $<0.001$ & $41(5.1)$ & $46(5.7)$ & 0.582 \\
\hline $\begin{array}{l}\text { Previous history of } \\
\text { TIA, stroke }\end{array}$ & $378(38.2)$ & $241(19.8)$ & $<0.001$ & $233(29.2)$ & $235(29.2)$ & 0.913 \\
\hline $\mathrm{CHA}_{2} \mathrm{DS}_{2}$-VASc score & $3.34 \pm 1.9$ & $3.5 \pm 1.7$ & 0.019 & $3.4 \pm 1.8$ & $3.3 \pm 1.8$ & 0.183 \\
\hline \multicolumn{7}{|l|}{ Laboratory findings } \\
\hline $\mathrm{Hs}-\mathrm{CRP}, \mathrm{mg} / \mathrm{dL}^{\dagger}$ & $0.2(0.1-0.5)$ & $0.59(0.2-2.3)$ & 0.002 & $1.24(0.2-3.4)$ & $0.4(0.1-2.3)$ & 0.028 \\
\hline NT-proBNP, pg/mL ${ }^{\dagger}$ & $\begin{array}{c}783.0 \\
(283.9-1913.3)\end{array}$ & $\begin{array}{c}2065.5 \\
(1032.3-3711.3)\end{array}$ & $<0.001$ & $\begin{array}{c}1005.4 \\
(337.4-2036.8)\end{array}$ & $\begin{array}{c}2992.5 \\
(1729.8-4253.8)\end{array}$ & $<0.001$ \\
\hline Creatinine, $\mathrm{mg} / \mathrm{dL}^{*}$ & $1.0 \pm 0.9$ & $0.9 \pm 0.6$ & 0.326 & $0.95 \pm 0.5$ & $0.96 \pm 0.6$ & 0.323 \\
\hline $\mathrm{CCl}, \mathrm{mL} / \mathrm{min}^{\dagger}$ & $83.8(67.3-93.9)$ & $73.2(58.7-97.4)$ & $<0.001$ & $87.0(71.8-110.9)$ & $85.4(66.1-107.4)$ & 0.054 \\
\hline
\end{tabular}

*Comparison made using chi-square test. ${ }^{\dagger}$ Median (25\% to $75 \%$ percentiles); comparison made using Mann-Whitney test. CCl: creatinine clearance, EF: left ventricular ejection fraction, HF: heart failure, Hs-CRP: high sensitivity C-reactive protein, MI: myocardial infarction, NT-proBNP: N-terminal pro-B-type natriuretic peptide, PS: propensity scores, TIA: transient ischemic attack.

der, age, past medical history, smoking and $\mathrm{CHA}_{2} \mathrm{DS}_{2}$-VASc scores. Also, there was no difference in the serum level of creatinine and creatinine clearance (Table 1 ).

\section{Clinical outcomes between the warfarin vs. the Rivaroxaban treatment groups: before PS matching}

There was no significant difference in the rate of total thromboembolic events between rivaroxaban and warfarin groups (Table 2). However, stroke rates were significantly lower in the rivaroxaban group [Hazard ratio (HR) $0.55,95 \%$ confidence interval (CI) 0.31-0.97, $\mathrm{p}=0.039$ ] which was mainly derived by a lower rate of hemorrhagic stroke [HR 0.22, 95\% CI 0.06-0.79, p=0.020]. Major bleeding events were lower in the rivaroxaban group [HR 0.51 , 95\% CI 0.31-0.85, $\mathrm{p}=0.009]$. In the subgroup analysis of major bleeding causes, mucosal bleeding [HR 0.56, 0.32-0.98, $\mathrm{p}=0.040$ ], and intracranial bleeding [HR $0.07,95 \% \mathrm{CI}$ $0.10-0.57, \mathrm{p}=0.013$ ] incidences were significantly lower in the rivaroxaban group than the warfarin group with no differences in the event rate of GI bleeding (Table 2).

Event rates of cardiac death, and MI were not different between the warfarin and rivaroxaban groups. However, all-cause death [HR 0.30, 95\% CI 0.16-0.55, p<0.001] and any bleeding events [HR 0.72, 95\% CI 0.57-0.91, $\mathrm{p}=0.006$ ] were significantly lower in the rivaroxaban group than the warfarin group.

Primary NCB was better in the rivaroxaban group than the warfarin group [HR 0.58, 95\% CI 0.42-0.81, $\mathrm{p}=0.002$ ]. However, there was no difference in secondary NCB be- tween two groups (Table 2).

\section{Clinical outcomes between the warfarin vs. the Rivaroxaban treatment groups: after PS matching}

After propensity score matching, there was no significant difference in the rate of total thromboembolic events between the rivaroxaban and the warfarin groups [HR 0.69, 95\% CI 0.37-1.32, Table 2, Fig. 1A, log-rank $\mathrm{p}=0.263$ ]. However, hemorrhagic stroke was still significantly lower in the rivaroxaban group than the warfarin group [HR 0.19, 95\% CI 0.04-0.85, $\mathrm{p}=0.030$ ]. There was no event of TIA in both groups (Table 2 ).

Major bleeding events were significantly lower in the rivaroxaban group [4.7\% vs $1.7 \%$, HR $0.41,95 \%$ CI $0.22-0.76$, $\mathrm{p}=0.004$, Table 2, Fig. 1B, log-rank $\mathrm{p}=0.003$ ]. There was no difference in the events rate of GI bleeding, and mucosal bleeding. There was no event of intracranial bleeding in the rivaroxaban group, whereas there were 13 occurrence in the warfarin group even though it was not statistically significant [HR 0.02, 95\% CI 0.00-1.45, p=0.075] (Table 2).

Event rates of cardiac death, and MI were not different between the warfarin and rivaroxaban groups. However, all-cause death [HR 0.31, 95\% CI 0.15-0.64, $\mathrm{p}=0.002$ ] and any bleeding events [HR 0.74, 95\% CI 0.56-0.97, p=0.032] were lower in the rivaroxaban group compared to warfarin.

Primary NCB was significantly better in the rivaroxaban group [HR 0.54, 95\% CI 0.36-0.81, p=0.003, Fig. 2A, $\log$-rank $\mathrm{p}=0.002]$. Secondary NCB was also significantly better in the rivaroxaban group [HR 0.62, 95\% CI 0.40-0.98, 


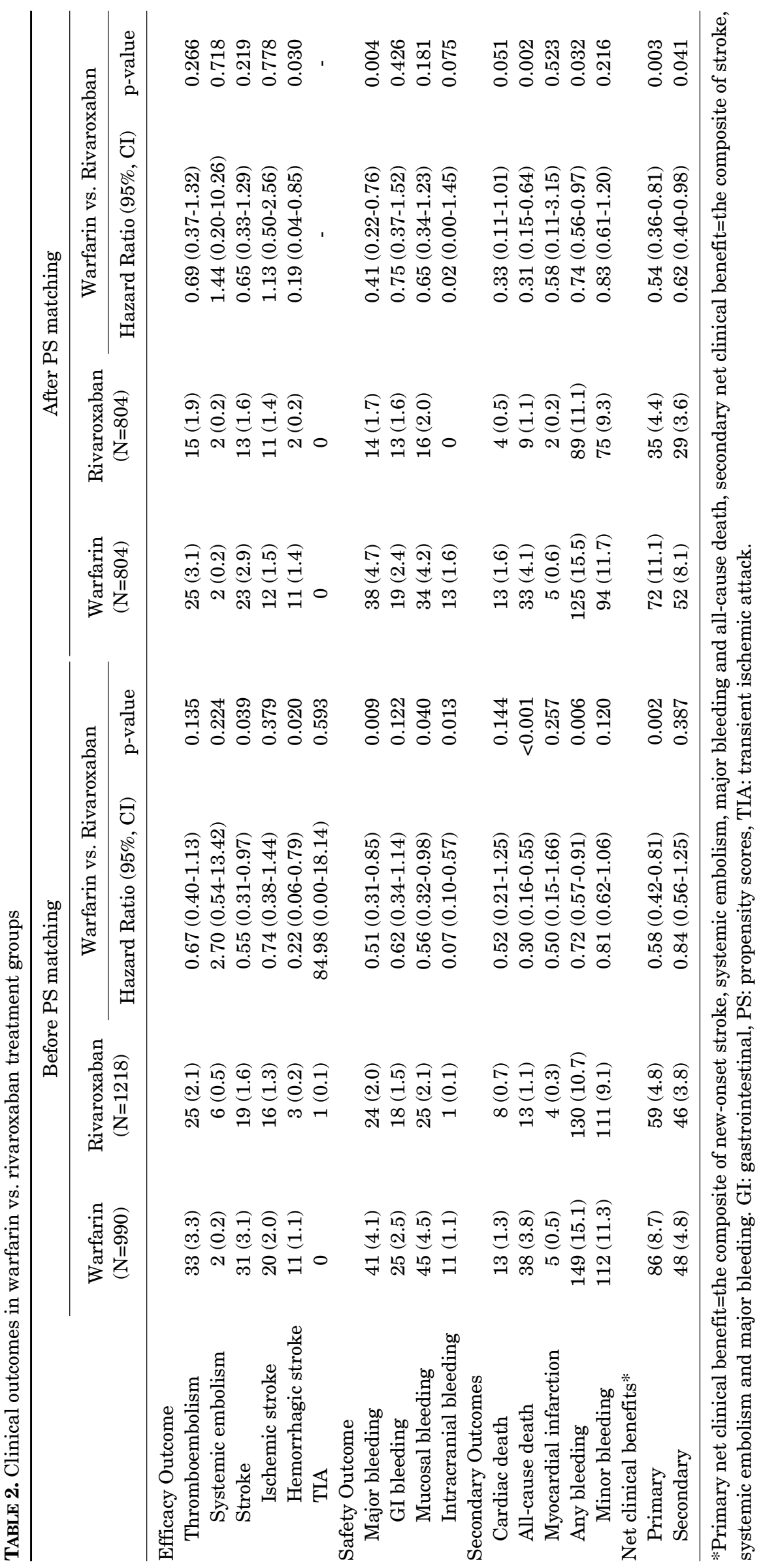


A

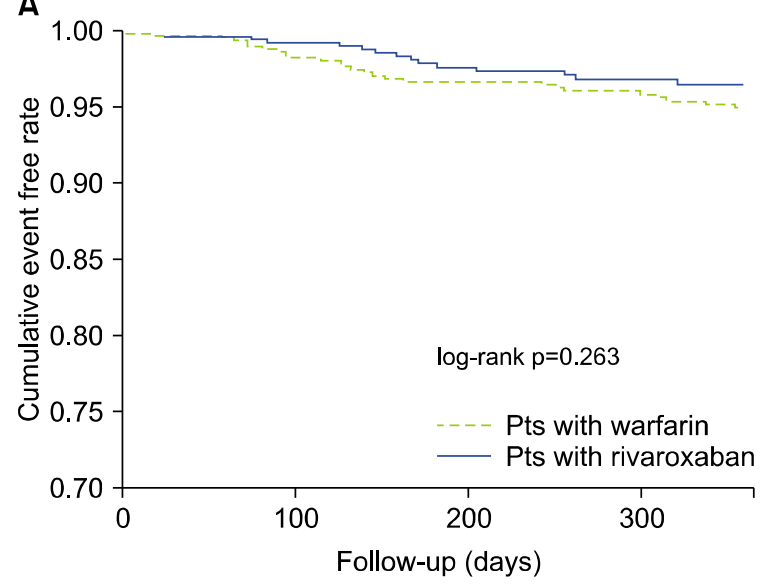

B

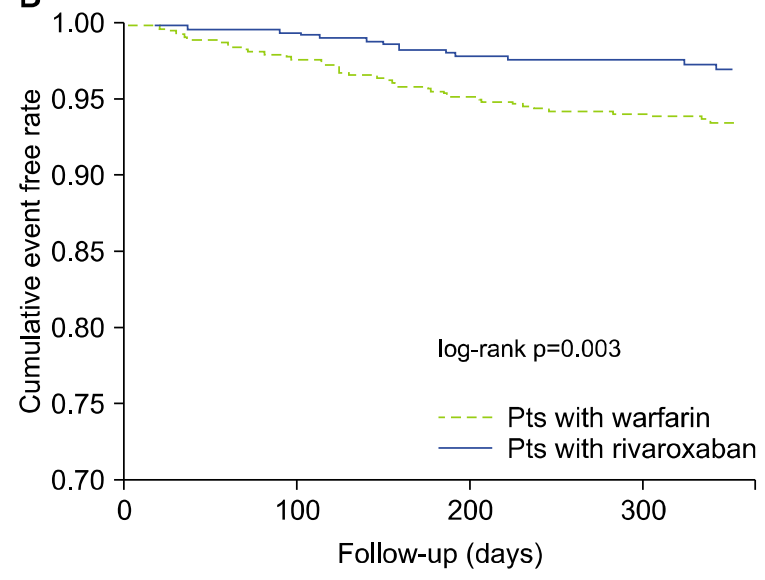

FiG. 1. (A) Kaplan-Meier estimation for stroke/systemic embolism between patients with warfarin and rivaroxaban, (B) Kaplan-Meier estimation for major bleeding between patients with warfarin and rivaroxaban.

A

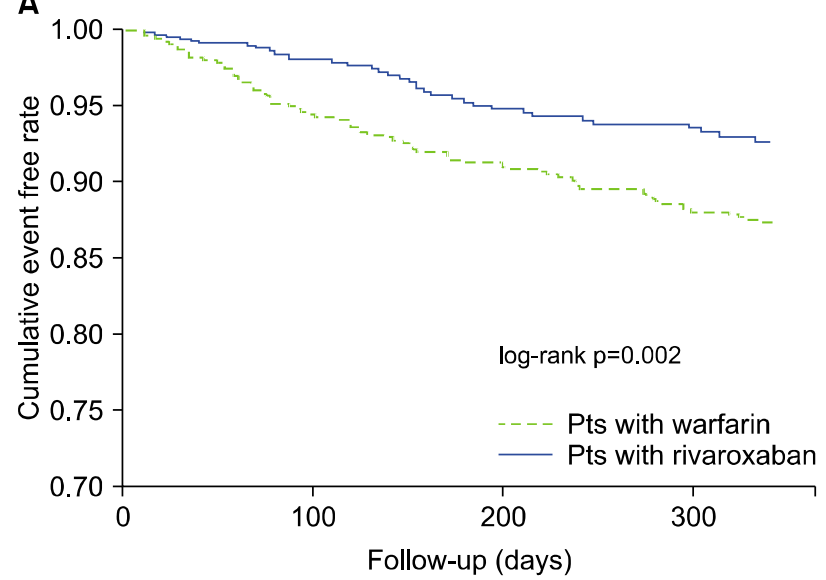

B

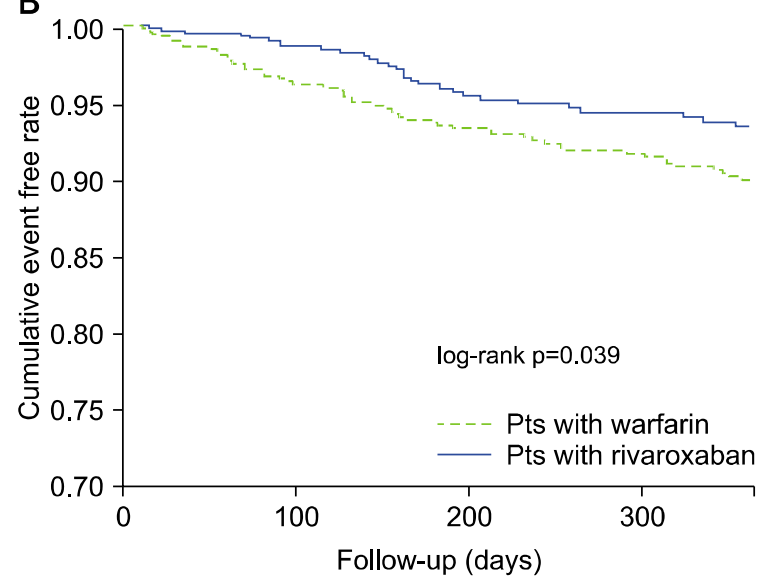

FiG. 2. (A) Kaplan-Meier estimation for primary net clinical benefit between patients with warfarin and rivaroxaban, (B) Kaplan-Meier estimation for secondary net clinical benefit between patients with warfarin and rivaroxaban.

$\mathrm{p}=0.041$, Fig. $2 \mathrm{~B}, \log$-rank $\mathrm{p}=0.039]$.

\section{Clinical outcome according to rivaroxaban dosage}

There were no differences in the risks of ischemic stroke, hemorrhagic stroke and systemic embolism between rivaroxaban $20 \mathrm{mg}$ versus (vs.) warfarin, or rivaroxaban $15 \mathrm{mg}$ vs. warfarin (Table 3 ). Major bleeding events were significantly lower in the rivaroxaban $20 \mathrm{mg}$ group [HR 0.35 , 95\% CI 0.15-0.82, $\mathrm{p}=0.016$ ] than warfarin (Table 3). Although major bleeding events in rivaroxaban $15 \mathrm{mg}$ were lower than the warfarin group, those were not statistically significant [HR $0.47,95 \%$ CI $0.22-1.01, \mathrm{p}=0.052$ ]. There were no differences in cardiac death, MI, any bleeding rates between rivaroxaban $20 \mathrm{mg}$ vs. warfarin, or rivaroxaban $15 \mathrm{mg}$ vs. warfarin. All-cause death was significantly lower in the rivaroxaban $20 \mathrm{mg}$ group than the warfarin group [HR 0.07, 95\% CI 0.01-0.51, $\mathrm{p}=0.009$ ]. Primary [HR 0.36, 95\% CI 0.20-0.67, $\mathrm{p}=0.001$ ] and secondary NCBs [HR 0.47, $95 \%$ CI 0.24-0.89, $\mathrm{p}=0.021]$ were lower in the rivaroxaban $20 \mathrm{mg}$ group than warfarin (Table 3 ). There were no differ- ences in primary and secondary NCBs between the rivaroxaban $15 \mathrm{mg}$ and warfarin groups.

\section{DISCUSSION}

The main finding of the present study was that rivaroxaban had similar efficacy to prevent thromboembolism, whereas it reduced hazards of major bleeding with better composite clinical outcomes compared to warfarin in Korean AF patients. No significant differences were observed between rivaroxaban and warfarin in terms of any bleeding subtypes including GI bleeding and mucosal bleeding. However, incidence of intracranial bleeding in the warfarin group was $1.6 \%$ while the rivaroxaban group was none.

At first, large randomized controlled trials demonstrated that rivaroxaban had non-inferiority of stroke prevention and there was no significant difference in major bleeding risk. ${ }^{6}$ It only decreased the risk of intracranial hemorrhage event. These were somewhat different results 


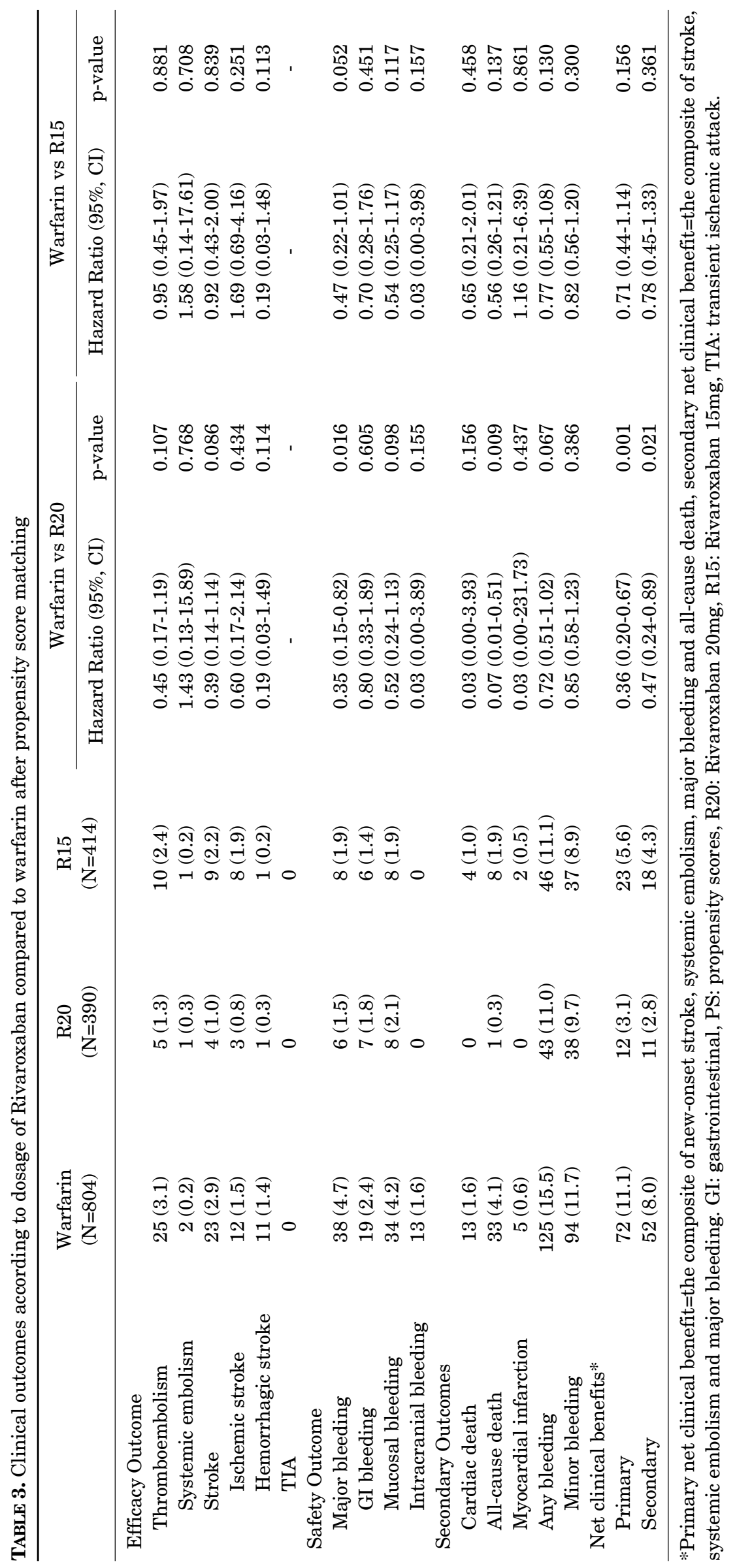


from consequent real-world studies and also different from our study results. ${ }^{8,15}$ In those studies, NOACs were better able to reduce major bleeding risk as well as being comparably effective at preventing strokes and thromboembolisms with warfarin. Consistent with previous real world studies, the present study also revealed rivaroxaban significantly lowered the major bleeding risk and all-cause mortality, although difference in stroke prevention efficacy was not statistically significant between rivaroxaban and warfarin. In addition, the present study showed the frequency of intracranial hemorrhage was definitively low, which was consistent with previous RCTs although it was not statistically elucidated since the event has not occurred in even one case in the rivaroxaban group.

The largest global real world studies, which have revealed clinical uses of rivaroxaban, have been conducted in multi countries, mostly among the western population. ${ }^{14}$ The patients of that study had a few different baseline characteristics, which included a greater incidence of hypertension, myocardial infarction, heart failure, and a lower incidence of diabetes mellitus, and prior history of stroke/ systemic embolism from present study. Our study patients had higher $\mathrm{CHA}_{2} \mathrm{DS}_{2}$-VASc scores compared to that study (3.3 \pm 1.8 vs. $2.0 \pm 1.3$ ). It demonstrated $1.9 \%$ of major bleeding, $12.9 \%$ of non-major bleeding and $0.8 \%$ of stroke/systemic embolism rates. Those incidences were similar with the present study which showed $1.7 \%$ of major bleeding rates and $11.1 \%$ of any non-significant bleeding rates. However, the present study found more frequent incidence of stroke/systemic embolism.

In terms of bleeding, Asian patients are more prone to suffer from major bleeding than Western patients. It has been accounted for by ethnic or racial differences in previous studies. ${ }^{10}$ This tendency reflected Japanese guidelines for anticoagulant therapy in patients with $\mathrm{AF}$, recommended an optimal range of INR to be 1.6-2.6 in patients aged over $70 .{ }^{19}$ As such, safety concerns have been major obstacles to manage AF patients. Accompanied with the fact that NOACs decreased the risk of major bleeding risk including intracranial hemorrhage, our study also advocated that rivaroxaban would be better choice for Asian AF patients who have higher risk of intracranial bleeding. This predilection was consistent with another Korean previous study. ${ }^{20}$ In that study, NOACs also significantly decreased the rate of major bleeding risk, but efficacy outcomes, like stroke prevention in the NOACs group, was similar with the warfarin group. This trend is a bit different compared to real world studies in western population. And these unique features support the greater benefits of NOACs in the Asian population.

The present study demonstrated bleeding events were higher in the rivaroxaban $15 \mathrm{mg}$ group than the rivaroxaban $20 \mathrm{mg}$. Firstly, the number of total patients was relatively small. Therefore, the event rate was also small, which demands cautious interpretation. The second reason is the so called off-label dose. Many Asian physicians are afraid of bleeding events because Asian patients with anticoagulation are prone to bleeding compared with Western population. Therefore, they have a tendency to prescribe a lower dose of NOACs without any indication. ${ }^{7}$ It was possible for patients who have clinically higher bleeding risk without dose reduction indication might be distributed into the rivaroxaban $15 \mathrm{mg}$ group in the present study. This predilection also could be found in global, real-world studies for Asian patients, which also demonstrated higher bleeding events in the rivaroxaban $15 \mathrm{mg}$ than the 20 mg group. ${ }^{21}$

There has been worry about relatively higher bleeding risks of rivaroxaban compared with other NOACs. ${ }^{8}$ Oncedaily doses of rivaroxaban would be expected to achieve higher peak and lower trough serum levels than a twicedaily dose. This tendency might affect the higher bleeding events in the rivaroxaban group than for the other NOACs. However, there has been no study which directly compares the bleeding events between rivaroxaban and the other NOACs. Also, bleeding events in Korean patients with rivaroxaban were consistently lower than warfarin. ${ }^{8,16,20} \mathrm{Be}-$ fore comparison of clinical outcomes between rivaroxaban and the other NOACs, differences of baseline clinical characteristics of enrolled patients should be considered. The mean $\mathrm{CHADS}_{2}$ score was highest in The Rivaroxaban Once Daily Oral Direct Factor Xa Inhibition Compared with Vitamin K Antagonism for Prevention of Stroke and Embolism Trial in Atrial Fibrillation (ROCKET AF) trial compared to the other NOACs' phase 3 randomized clinical trials. ${ }^{6} \mathrm{~A}$ high $\mathrm{CHADS}_{2}$ score predisposed patients to higher risk of stroke and bleeding. Therefore, a simple comparison of clinical outcomes results in apparent higher bleeding risk in patients with rivaroxaban. Recently published studies with rivaroxaban consistently showed lower $\mathrm{CHADS}_{2}$ scores and lower events rates of major bleeding in the real-world clinical field compared with the ROCKET-AF trial. $^{14,21,22}$

The present study has several limitations. First, this study was based on a retrospective analysis in a single center. Therefore, a selection bias may impact study's internal validity. Even though propensity score matching can make the cohorts have the similar baseline characteristics, only measurable variables were matched. Thus, residual confounding factors cannot be excluded and possibly might remain. Multicenter prospective trials are needed to reflect comprehensive real world clinical circumstances. Second, the sample size was relatively small compared to previous randomized controlled trials or global real world studies. Thus, event rates were also not that frequent. Nevertheless, the present study was performed in Asian patients, particularly, in Korean patients. To our best knowledge, this is the $1^{\text {st }}$ study representing Korean clinical situations so far. Third, the warfarin group did not investigate INR values which indicated the quality of the warfarin treatment. Therefore, we could not make sure whether all patients who took warfarin remained in the time in therapeutic range (TTR) or not. Further studies are needed to include the patients have the optimal TTR using 
warfarin.

In conclusion, in patients with non-valvular AF, rivaroxaban had similar efficacy to warfarin in Korean real world practice. However, rivaroxaban had better safety and net clinical benefits compared with warfarin in the 1 year follow-up. Although real-world data is reassuring, further large multicenter trials are needed to confirm that rivaroxaban is the better choice in Korean AF patients.

\section{CONFLICT OF INTEREST STATEMENT}

None declared.

\section{REFERENCES}

1. Roger VL, Go AS, Lloyd-Jones DM, Benjamin EJ, Berry JD, Borden WB, et al; American Heart Association Statistics Committee and Stroke Statistics Subcommittee. Heart disease and stroke statistics--2012 update: a report from the American Heart Association. Circulation 2012;125:e2-220.

2. Kirchhof P, Benussi S, Kotecha D, Ahlsson A, Atar D, Casadei B, et al. 2016 ESC Guidelines for the management of atrial fibrillation developed in collaboration with EACTS. Europace 2016;18:1609-78.

3. Granger CB, Alexander JH, McMurray JJ, Lopes RD, Hylek EM, Hanna M, et al. Apixaban versus warfarin in patients with atrial fibrillation. N Engl J Med 2011;365:981-92.

4. Connolly SJ, Ezekowitz MD, Yusuf S, Eikelboom J, Oldgren J, Parekh A, et al. Dabigatran versus warfarin in patients with atrial fibrillation. N Engl J Med 2009;361:1139-51.

5. Giugliano RP, Ruff CT, Braunwald E, Murphy SA, Wiviott SD, Halperin JL, et al. Edoxaban versus warfarin in patients with atrial fibrillation. N Engl J Med 2013;369:2093-104.

6. Patel MR, Mahaffey KW, Garg J, Pan G, Singer DE, Hacke W, et al. Rivaroxaban versus warfarin in nonvalvular atrial fibrillation. N Engl J Med 2011;365:883-91.

7. Chan YH, See LC, Tu HT, Yeh YH, Chang SH, Wu LS, et al. Efficacy and safety of apixaban, dabigatran, rivaroxaban, and warfarin in Asians with nonvalvular atrial fibrillation. J Am Heart Assoc 2018;7:e008150.

8. Joung B. Real-world data and recommended dosage of non-vitamin $\mathrm{K}$ oral anticoagulants for Korean patients. Korean Circ J 2017;47:833-41.

9. Lopes RD, Guimarães PO, Kolls BJ, Wojdyla DM, Bushnell CD, Hanna M, et al. Intracranial hemorrhage in patients with atrial fibrillation receiving anticoagulation therapy. Blood 2017;129:
2980-7.

10. Shen AY, Yao JF, Brar SS, Jorgensen MB, Chen W. Racial/ethnic differences in the risk of intracranial hemorrhage among patients with atrial fibrillation. J Am Coll Cardiol 2007;50:309-15.

11. Li YG, Lee SR, Choi EK, Lip GY. Stroke prevention in atrial fibrillation: focus on Asian patients. Korean Circ J 2018;48:665-84.

12. Lip GY, Wang KL, Chiang CE. Non-vitamin K antagonist oral anticoagulants (NOACs) for stroke prevention in Asian patients with atrial fibrillation: time for a reappraisal. Int J Cardiol 2015; 180:246-54

13. Wang KL, Lip GY, Lin SJ, Chiang CE. Non-vitamin K antagonist oral anticoagulants for stroke prevention in Asian patients with nonvalvular atrial fibrillation: meta-analysis. Stroke 2015;46: 2555-61.

14. Camm AJ, Amarenco P, Haas S, Hess S, Kirchhof P, Kuhls S, et al. XANTUS: a real-world, prospective, observational study of patients treated with rivaroxaban for stroke prevention in atrial fibrillation. Eur Heart J 2016;37:1145-53.

15. Beyer-Westendorf J, Camm AJ, Coleman CI, Tamayo S. Rivaroxaban real-world evidence: validating safety and effectiveness in clinical practice. Thromb Haemost 2016;116(Suppl 2):S13-23.

16. Lee KH, Park HW, Cho JG, Yoon NS, Kim SS, Kim MR, et al. Comparison of non-vitamin $\mathrm{K}$ antagonist oral anticoagulants and warfarin on clinical outcomes in atrial fibrillation patients with renal dysfunction. Europace 2015;17 Suppl 2:ii69-75.

17. Lee KH, Park HW, Lee N, Hyun DY, Won J, Oh SS, et al. Optimal dose of dabigatran for the prevention of thromboembolism with minimal bleeding risk in Korean patients with atrial fibrillation. Europace 2017;19(Suppl 4):iv1-9.

18. Schulman S, Kearon C. Definition of major bleeding in clinical investigations of antihemostatic medicinal products in non-surgical patients. J Thromb Haemost 2005;3:692-4.

19. JCS Joint Working Group. Guidelines for pharmacotherapy of atrial fibrillation (JCS 2013). Circ J 2014;78:1997-2021.

20. Cha MJ, Choi EK, Han KD, Lee SR, Lim WH, Oh S, et al. Effectiveness and safety of non-vitamin $\mathrm{K}$ antagonist oral anticoagulants in Asian patients with atrial fibrillation. Stroke 2017; 48:3040-8.

21. Kim YH, Shim J, Tsai CT, Wang CC, Vilela G, Muengtaweepongsa S, et al. XANAP: a real-world, prospective, observational study of patients treated with rivaroxaban for stroke prevention in atrial fibrillation in Asia. J Arrhythm 2018;34:418-27.

22. Coleman CI, Antz M, Bowrin K, Evers T, Simard EP, Bonnemeier $\mathrm{H}$, et al. Real-world evidence of stroke prevention in patients with nonvalvular atrial fibrillation in the United States: the REVISITUS study. Curr Med Res Opin 2016;32:2047-53. 